\title{
Transplant Critical Care: Is There A Need for Sub-specialized Units? - A Perspective
}

\author{
Sujit Vijay Sakpal1,2,3*, Suresh Kumar Agarwal4 , Hector Saucedo-Crespo1,2, Christopher \\ Auvenshine ${ }^{1,2}$, Robert N. Santella1,3, Steven Donahue², Jeffery Steers ${ }^{1}$ \\ ${ }^{1}$ Avera McKennan Hospital \& University Health Center: Avera Medical Group Transplant \& Liver Surgery. Sioux Falls, \\ South Dakota, USA \\ 2 Department of Surgery, University of South Dakota. Sioux Falls, South Dakota, USA \\ 3 Department of Internal Medicine, University of South Dakota. Sioux Falls, South Dakota, USA \\ 4 Division of Acute Care, Trauma, Surgical Critical Care. Department of Surgery. Duke University. Durham, North \\ Carolina, USA
}

\begin{abstract}
The critical care involved in solid-organ transplantation (SOT) is complex. Pre-, intra- and post-transplant care can significantly impact both - patients' ability to undergo SOT and their peri-operative morbidity and mortality. Much of the care necessary for medical optimization of end-stage organ failure (ESOF) patients to qualify and then successfully undergo SOT, and the management of peri-operative and/or long-term complications thereafter occurs in an intensive care unit (ICU) setting. The current literature specific to critical care in abdominal SOT patients was reviewed. This paper provides a contemporary perspective on the potential multifactorial advantages of sub-specialized transplant critical care units in providing efficient, comprehensive, and collaborative multidisciplinary care.
\end{abstract}

Keywords: solid-organ transplantation, abdominal organ transplantation, critical care medicine

Received: 15 June 2018 / Accepted: 15 July 2018

\section{INTRODUCTION}

Knowledge and technology have transformed the practice of medicine, and both with the need to optimize outcomes have been the driving force in creating subspecialties in virtually every realm of clinical medicine. Critical care medicine is no exception with the consequent evolution of sub-specialized units in the areas of pediatrics and neonatology, cardiology and cardiac surgery, and neurology and neurosurgery [1]. Patients with end-stage organ failure (ESOF), particularly those in need of, or those who have undergone solid-organ transplantation (SOT), represent a unique patient population. Complexities in the management of SOT patients pose challenges to the healthcare team at every level. The critical care of SOT patients, both in the pre- and post-transplant phase, is essential. It impacts on their potential candidacy for donated organs and subsequently influences their post-transplant morbidity, mortality, and allograft survival [2]. With pre-transplant ESOF, an extensive, complicated surgical procedure, unique post-operative management of the recipient (allograft included) and careful balance of immunosuppression, it is imperative that critical care rendered to the transplant patient has a multidisciplinary approach inclusive of transplant physicians and skilled intensivists.

\section{Challenges in TRANSPLANT Patients}

Patients with impending or established single- or multi-organ failure can be defined as critically ill. Patients awaiting SOT and transplant recipients often drastically decompensate when complications arise. Review articles by Findlay et al. and Razonable et al. outline topics in critical care management of unique complications, encountered to varying degrees, in SOT patients $[3,4]$.

\section{PRE-TRANSPLANT PHASE}

Successful management of patients with ESOF requires an understanding of their pathophysiology and the complications that may ensue. It is not uncommon to 
encounter bouts of hepatic encephalopathy (HE) and spontaneous bacterial peritonitis (SBP) in potential liver recipients, or catheter-related multi-drug resistant (MDR) sepsis with disseminated infection in patients with end-stage renal disease (ESRD) with challenging dialysis access or those with intestinal failure dependent on parenteral nutrition. Complications such as porto-pulmonary hypertension ( $\mathrm{PPH}$ ) and hepatopulmonary syndrome (HPS) in end-stage liver disease (ESLD) patients compromise cardiovascular physiology, ventricular function and respiratory mechanics which may threaten transplantation [5]. Renal failure, acute or chronic, in ESLD patients necessitates early recognition, differentiation from hepatorenal syndrome (HRS), and timely treatment that may involve renal replacement therapy (RRT). In wait-listed ESLD patients, progressive deterioration of renal function or prolonged RRT demands periodic re-evaluation for multi-organ transplantation. While complications, as mentioned above, dictate patients' survival, they pose a constant threat to their transplant eligibility and may jeopardize their candidacy for life-saving organ offers. Thus, it is essential that one understands the pursuit of aggressive therapy in these patients to enable them to receive SOT and thereby heal.

In addition to treating complications, pre-transplant medical optimization of ESOF patients is a vital task. A concerted, multidisciplinary approach is key to maintaining a patient's eligibility for transplantation. For example, orchestrating collaborative therapeutic strategies with neurocritical care intensivists and nephrologists with regards to volume status, and hepatologists and infectious disease (ID) specialists, is critical for preventing a fatal outcome from elevated intracranial pressure (ICP) in patients with acute liver failure (ALF) [6,7]. Similarly, patients suffering from $\mathrm{PPH}$ and HPS require dedicated pharmacological therapy with the keen management of fluid-volume status in accord with a pulmonologist.

\section{DOST-TRANSPLANT PHASE}

Cardiovascular disease and related events are the leading cause of morbidity, graft loss, and mortality in kidney transplant recipients [8]. Optimizing recipient physiology following SOT yields the most significant chance of allograft function and overall survival. A multivariable balancing act inclusive of continuous fluid-volume status adjustments, customized antico- agulation if and when necessary, and maintenance of adequate tissue perfusion is vital in the restoration of physiological homeostasis and prevention of allograft thrombosis. At the time of obtaining consent before transplantation, patients are informed of the "big operation, big incision, big pain, big recovery, and possible big complications." More often than expected, transplant recipients encounter various acute or chronic complications.

A multitude of different bacterial, viral and fungal infections may plague transplant recipients requiring aggressive testing methods and alternative therapeutic strategies. Immediately post-transplant, recipients may suffer from wound infection, C. difficile colitis, urinary tract infection (UTI), or ventilator-associated events (VAEs) from prolonged mechanical ventilation. Bile leak(s) or anastomotic ductal stenosis may progress to cholangitis, bacteremia, sepsis and shock in liver recipients. Alike, urinoma(s) and ureteral stricture(s) may develop infectious complications in kidney recipients. Furthermore, a variety of opportunistic and late post-transplant infections may cause transplant recipients to be admitted to the intensive care unit (ICU). Frequently, transplant recipients (including kidney) sustain acute kidney injury (AKI) and may even require RRT. This may exacerbate cardiorespiratory insufficiency or failure especially in recipients with preexisting pulmonary complications of cirrhosis or those with suboptimal cardiac or ventricular function. That said, very often we witness liver transplantation to be the therapy, cause for the cure of HRS, HPS, PPH, cerebral edema, hyperdynamic circulation, multifactorial coagulopathy, and Kwashiorkor malnutrition [9]. Such remarkable transformations are mainly limited to the field of SOT.

The incidence and severity of post-transplant complications are remarkably high in intestinal transplant (IT) recipients. In this group of SOT recipients - micronutrient deficiencies commonly occur due to the gradual allograft adaptation. Acute and/or chronic allograft rejection may manifest with increased stomal output and consequent AKI, and sepsis secondary to bacterial translocation [10,11]; and graft-versus-host disease (GVHD) has an incidence of $7 \%$ due to the presence of a robust intestinal lymphatic system which may present with hepatic dysfunction and bone marrow depression [12].

A widening gap between the supply of allografts appropriate for transplantation and the continu- 
ous increase in demand concerning patients awaiting transplant has prompted aggressive donor utilization. Simultaneously, increasingly moribund and decompensated recipients are undergoing transplantation. Thus, early identification and appropriate management of poorly functioning grafts have become a critical aspect of post-transplant care $[4,13]$. Hepatic artery (HAT) or portal venous thrombosis (PVT), and venous outflow or biliary obstruction requires timely recognition and therapy to save or improve liver allograft function. Similarly, any suspected vascular compromise following renal or pancreatic transplantation necessitates swift diagnosis and treatment. Also, modifying, tailoring and balancing immunosuppressive regimen in SOT recipients has a pivotal role in combating complications inclusive of rejection or infection.

\section{IS THERE A NEED FOR TRANSPLANT CRITI- CAL CARE?}

In 2012 , of the total 5,731 adult liver transplants performed in the United States, $17.9 \%(1,028)$ occurred in recipients with a Model for End-stage Liver Disease (MELD) score $>35$ [14]. Before transplantation, $723(12.6 \%)$ recipients were hospitalized in the ICU of which 403 (7\%) had been on life support, and 192 (3.4\%) were listed as status 1 . Early post-transplant tracheal extubation is safe and suggests efficient resource use without significant reduction in ICU or hospital length of stay (LOS) in selected liver transplant patients $[15,16]$. However, despite attempts to fast-track care and avoid an ICU stay, $45.7 \%$ of orthotropic liver transplant (OLT) patients require immediate ICU stay [17].

Infrequent compared to liver, $6.6 \%(452 / 6,819)$ of kidney transplant patients demonstrated the need for ICU admission in a study by Canet et al. [18]. Fortyfour percent (200) of these patients were admitted for acute respiratory failure from bacterial pneumonia (35.5\%), cardiogenic pulmonary edema $(24.5 \%)$ and extrapulmonary acute respiratory distress syndrome (ARDS) (15.5\%); and almost half of these patients required either, or a combination thereof, mechanical ventilation, vasopressors, and RRT. Other studies have shown that up to $20 \%$ of renal transplant recipients require escalation of care from the ward to ICU and higher mortality among kidney recipients requiring ICU than the general ICU population $(42.6 \%$ vs $30 \%)[19,20]$. Only $1.4 \%$ of renal transplant recipients are readmitted to the ICU but up to $35 \%$ of first time successful OLTs return to the ICU primarily for infectious or septic complications (51\%), cardiopulmonary dysfunction (35\%) and graft failure (22\%) [21]. Most infrequent of the abdominal SOTs, data from 165 ITs performed at a single center revealed a mean duration of 16+/- 23 ICU days post-transplantation [10]. These aforementioned figures underestimate the actual use of the ICU by SOT patients because it does not include those who get admitted to the ICU with an acute illness but recover, those who die in the ICU without having their status upgraded, or those who get admitted to the ICU for a condition that results in their removal from the transplant wait-list [9]. With an increasing number of transplant candidates on the list and their extended length of time awaiting organs, increasing number of patients requiring ICU care before transplantation can be anticipated.

Thus, inception of sub-specialized transplant critical care units at moderate-to-high-volume centers have the potential to have a multifold impact - the welfare of individual patient(s) and allograft(s), development of the multi-organ transplant program, and further the understanding of modern surgical critical care of complex SOT patients.

\section{Patients First, AND The Allograft TOO!}

Specialized care is an absolute necessity to combat complexities that may occur in transplant candidates and recipients. In fact, candidacy for life-saving organs relies heavily on the critical management of extremely sick patients awaiting transplants. For patients who are not transplant candidates, the goal is the restoration of pre-ICU functional status, recognizing that ESOF is an incurable condition without transplantation [22]. For listed transplant patients, the goal is to provide a "window" of clinical stability when transplantation could be feasible. For patients who are possible transplant candidates, the goal is to provide a "window" of opportunity to complete a candidacy work-up. The key challenge is keeping this "window" open because recurrence of illness after resolution of the initial episode is common. Striving to achieve these "windows" of opportunity must be counterbalanced against potentially futile care and provision of false hope. In critically ill ESOF patients, deemed as transplant candidates, the following series of events must take place to achieve a good outcome: 
- resolution of acute illness,

- non-recurrence of another illness,

- completion of candidacy work-up, and

- a successful transplant operation at the availability of suitable organ(s).

Provision of post-operative care is a fundamentally shared responsibility of the multidisciplinary care team including subspecialty physicians, intensivists, and transplant surgeons who understand complex anatomy as well as the impact of the procedures they perform on the individual patient's physiology [2]. Post-operative allograft function or dysfunction reflects the interplay of donor factors, the recipient's acuity and the recipient's operative course [1]. It is not just monitoring of graft function that is vital, but early suspicion and recognition of graft dysfunction is critical. While implantable continuous Dopplers are being used for prompt detection of HAT post-liver transplantation, such devices still do not substitute the medical judgement and experience of skilled transplant surgeons in conjunction with critical care-trained physicians [10]. More importantly, rapid assessment of the recipient must be followed up with, if necessary, escalated care and definitive therapeutics implemented expeditiously sometimes to save an allograft. For example, elevated liver function tests (LFTs) post-OLT should prompt acquisition and interpretation of liver Doppler images with immediate angiographic or operative intervention as necessary.

Acute and chronic pathophysiologic changes associated with SOT can cause substantial emotional distress and psychological stresses among this subset of patients. These stresses are accentuated in the ICU setting which could influence immediate and long-term outcomes of SOT. Thus, in addition to their medical needs, it is essential to recognize and address the psychological and psychiatric needs of these patients [23].

\section{- VOLUME EXPANSION, QUALITY IMPROVE- MENT \& RESOURCE UTILIZATION}

Volume expansion continues to be the biggest challenge in the field of SOT. Despite significant scientific advancements in the process of SOT over the past five decades, the stagnant supply of quality organs has been unable to match the ongoing demand of allografts. In the United States, approximately only a quarter of the current wait-listed candidates for all organ types $(114,561)$ have been transplanted annually over the past decade $(27,764$ - 34,772) [24]. Thus, to sustain or better outcomes one has to think outside the box.

Three decades ago, up to $40 \%$ of all deaths in liver transplant recipients occurred in the ICU, mostly from infectious causes [25]. Comprehensive understanding of SOT physiology and pathophysiology, and advancements in medicine - antibiosis, immunosuppression, diagnostic-therapeutic modalities in critical care - have effectively reduced the mortality among critically ill OLT recipients below the overall mortality rate for all ICU admissions (10.6\% versus 15\%) [21]. Effective critical care supports a transplant program's ability to consider high-risk donors and high-risk recipients, which expectantly would enhance the overall survival of the population of patients with ESOF $[1,26]$. This is where assurance of skillful, experienced, transplantspecific and critical care competent peri-operative management is essential to be able to:

- venture with suboptimal organ offers,

- cater to complex patients with multiple co-morbidities or high "MELDers", and

- produce consistent and improved results.

Hospitals incur substantial costs due to repetitive, redundant and frequently unnecessary physician consultations and diagnostic tests. Also, soaring healthcare costs cannot be neglected by the current state of affairs. Care rendered to patients post-transplant (or pretransplant) determines their morbidity, mortality and largely dictates outcomes, which is perceived as quality. Thus, emphasis must be on providing consistent, efficient and high-quality care to patients primarily in a high-volume setting to produce and sustain superior outcomes.

Undeniably, multidisciplinary care is central to the management of SOT patients. Invaluable input from experienced specialists in critical care, hepatology, nephrology, anesthesiology, ID, hematology, and pulmonology is essential for the successful care of SOT patients. Patients' intensive care team also includes, and is not limited to, specialized transplant nurses, advanced practice providers (APPs), pharmacists, psychologists, respiratory-physical-occupational therapists (RT, PT, OT), and social workers or case managers. Self-learning and continually educating team members is vital. Additionally, astute utilization of available resources and timely, relevant assistance from interdisciplinary colleagues should be the strategy for practical outcomes.

Allotment of physical space with dedicated resources solely for a SOT program is a considerable task. Its fea- 
sibility depends on multiple factors including administrative and financial support based on an institution's individualized funding capabilities, and the influx of ESOF patients referred for SOT evaluation. While it may be economically justifiable to "concentrate" space and resources in establishing a transplant-only ICU at high volume multi-organ SOT centers, the concept may prove to be inefficient and unjustified with regards to apt utilization of resources at low-to-moderate volume SOT centers. Differently, allocation of designated beds within an institution's existing ICUs (for example, 5 to 8 beds in a 24-bed medical or surgical ICU) with an option to "overflow" may be an equally competent alternative. Note, that we emphatically stress the value of leading a collaborative, multidisciplinary program which includes the aforementioned transplant-specialized clinicians and all members of the intensive care team. Regardless of the actual existence of a transplantonly ICU, an ambitious transplant leadership must strive to cultivate an intensive care team equipped with the "transplant ways" essential to efficiently manage ESOF patients, simultaneously and expeditiously enable assessment of patients' candidacy for SOT, and treat patients, either peri-operatively or those with long-term complications, following SOT.

While some ESOF patients do not qualify to become transplant candidates, some transplant recipients suffer and succumb to irreversible graft failure or fatal complications. For these patients, responsibly determining futility of care can be a challenge, yet it is essential to maintain communication within the multidisciplinary care team and with patients or their families to achieve early consensus on goals of care for dying transplant patients. Timely integration of palliative care alongside an aggressive, disease-focused, curative care must be accomplished in the ICU to improve the end-of-life care practice for patients in whom prolonging treatment is deemed futile [27]. Also, transfer of the terminally ill transplant patient to the ICU must be refrained to avoid the unnecessary utilization of resources, the provision of false hopes and expectations, and the indignities that occur when suffering and death take place in an inappropriate environment [9].

Hence, collaborative efforts between the transplant and ICU physicians and teams are essential to the management of the SOT patient. Creating a workforce of clinicians who have training in both specialties may help to synergize the care required for this complex patient population.

\section{model Creation, innovation}

The field of SOT has matured significantly over the last two decades. Rather than being complacent of the accomplishments and accept the status quo, this is the time to build, expand and innovate. Advancements in operative techniques and developments in immunosuppressive therapy have paved the way to perform more complex surgical procedures under challenging clinical scenarios. Hence, continually identifying areas in need of improvement is essential, and critical transplant care is one such area.

Design and establishment of a goal-directed, dedicated, sub-specialized ICU to cater to the critical needs of ESOF patients, either in need of an allograft or those who have received one (or more), will provide an opportunity to explore novel models in SOT patient care. Creation of a prospective database of critically ill transplant candidates and recipients will allow us to:

- expand understanding of complex pathologies in this unique class of patients,

- assess and improvise day-to-day performance, and

- modify current protocols and design new ones based on evolving evidence.

Such design-thinking makes for a robust approach, in combination with creativity, to perform a needsfinding exercise which can identify unmet needs and explore areas of opportunity.

It could be argued that surgeons without formal critical care training may not be ideal or rather wellequipped for managing complicated ventilator settings or pressors-ionotropes in hemodynamically labile patients. However, most abdominal transplant surgeons and in-training fellows receive a concentrated experience in the care of SOT patients including their ICU care [2]. That said, this may be an impetus for abdominal SOT fellowship training programs to expand their curriculum involving intensive care. For example, "routine" ICU procedures such as endotracheal intubation, advanced ventilator management, fiber optic bronchoscopy and point-of-care-ultrasonography (POCUS) should be considered for mandatory inclusions. As surgical training continues to evolve, the inclusion of an intensive-care specific curriculum in addition to the technical training that SOT fellows receive may provide a workforce that can straddle the worlds of transplant and intensive care. In addition, including a transplant-specific rotation during an intensive care 
fellowship - focusing on the needs of the SOT patient without learning the technical aspects - may assist with developing the workforce that is necessary for developing a transplant-specific intensive care model.

\section{CONCLUSION}

Incorporation of sub-specialized critical care units in the management of transplant patients has the potential to have a global impact. Under a transplant and intensive care physician collaborative leadership model synchronized, multidisciplinary and aggressive efforts are paramount to:

- maximize SOT in qualified potential recipients, and

- successfully combat probable complications and improve overall outcomes in allograft recipients.

\section{ABBREVIATIONS}

AKI, acute kidney injury

ALF, acute liver failure

ARDS, acute respiratory distress syndrome

ESLD, end-stage liver disease

ESOF, end-stage organ failure

ESRD, end-stage renal disease

GVHD, graft-versus-host disease

HAT, hepatic artery thrombosis

HE, hepatic encephalopathy

HPS, hepatopulmonary syndrome

HRS, hepatorenal syndrome

ICP, intracranial pressure

ICU, intensive care unit

ID, infectious disease

IT, intestinal transplant

LFTs, liver function tests

LOS, length of stay

MELD, model for end-stage liver disease

MDR, multi-drug resistant

OLT, orthotopic liver transplant

POCUS, point-of-care ultrasonography

PPS, portopulmonary syndrome

PVT, portal venous thrombosis

RRT, renal replacement therapy
SBP, spontaneous bacterial peritonitis

SOT, solid-organ transplantation

UTI, urinary tract infection

VAE, ventilator-associated event

\section{AUTHORSHIP}

Sujit Vijay Sakpal, MD: Study conception of design; drafting of manuscript; critical revision; review, analysis and interpretation of scientific literature

Suresh Kumar Agarwal, MD, FACS, FCCM: Study conception of design; critical revision

Hector Saucedo-Crespo, MD: Drafting of manuscript; review, analysis and interpretation of scientific literature

Christopher Auvenshine, DO, FACOS: Drafting of manuscript; review, analysis and interpretation of scientific literature

Robert N. Santella, MD, FACP: Drafting of manuscript; critical revision

Steven Donahue, BA, MSIV: Drafting of manuscript; review, analysis and interpretation of scientific literature

Jeffery Steers, MD, FACS: Drafting of manuscript; critical revision

\section{DISCLOSURES}

The authors of this manuscript state that there is no relevant, real or apparent financial interest or affiliation with the issues discussed in this article.

\section{DFUNING}

No outside funding was obtained for this project.

\section{DEFERENCES}

1. Niemann CU, Kramer DJ. Transplant critical care: standards for the intensive care of the patient with liver failure before and after transplantation. Liver Transpl. 2011;17:485-7.

2. Ranjan D, Klintmalm G, Roberts J, Members of Critical Care Task Force, ASTS. American Society of Transplant Surgeons' White Paper: Impact of Closed ICUs on Transplant Patients' Care. Am J Transplant. 2011;11:670-1.

3. Findlay JY, Fix OK, Paugam-Burtz $C$, et al. Critical care of the end-stage liver disease patient awaiting liver transplantation. Liver Transpl. 2011;17:496-510. 
Available online at: www.jccm.ro

4. Razonable RR, Findlay JY, O'Riordan A, et al. Critical care issues in patients after liver transplantation. Liver Transpl. 2011;17:511-27

5. Saner FH, Sotiropoulos GC, Radtke A, et al. Intensive care unit management of liver transplant patients: a formidable challenge for the intensivist. Transplant Proc. 2008;40:3206-8.

6. Trotter JF. Practical management of acute liver failure in the Intensive Care Unit. Curr Opin Crit Care. 2009;15:163-7.

7. Schilsky ML, Honiden S, Arnott L, Emre S. ICU management of acute liver failure. Clin Chest Med. 2009;30:71-87.

8. Lenten KL, Brennan DC, Schnitzler MA. Incidence and predictors of myocardial infarction after kidney transplantation. JASN. 2005;16:496-506

9. Plevak DJ, Findlay JY. Critical care issues in liver transplantation. Liver Transplantation. 2000;6: S2-4.

10. Abu-Elmagd K, Reyes J, Bond $G$, et al. Clinical intestinal transplantation: a decade of experience at a single centre. Ann Surg. 2001;234:404-16.

11. Ubesie AC, Cole CR, Nathan JD et al. Micronutrient deficiencies in pediatric and young adult intestinal transplant patients. Pediatr Transplant. 2013;17:638-45.

12. Wu G, Selvaggi G, Nishida $\mathrm{S}$ et al. Graft-versus-host disease after intestinal and multivisceral transplantation. Transplantation. 2011;91:219.

13. Plevak DJ, Southorn PA, Narr BJ, Peters SG. Intensive-care unit experience in the Mayo liver transplantation program: the first 100 cases. Mayo Clin Proc. 1989;64:433-45.

14. Annual Report of the US Scientific Registry for Transplant Recipients and the Organ Procurement and Transplantation Network: Transplant Data. 2012. http://srtr.transplant.hrsa. gov/annual_reports/2012/pdf/03_liver_13.pdf

15. Neelakanta G, Sopher M, Chan S et al. Early tracheal extubation after liver transplantation. J Cardiothorac Vasc Anesth. 1997;11:165-7.

16. Mandell MS, Lockrem J, Kelley SD. Immediate tracheal
The Journal of Critical Care Medicine 2018;4(3) • 89

extubation after liver transplantation: experience of two transplant centers. Anesth Analg. 1997;84:249-53.

17. Bulatao IG, Heckman MG, Rawal B, et al. Avoiding stay in the intensive care unit after liver transplantation: a score to assign location of care. Am J Transplant. 2014;14:2088-96.

18. Canet E, Osman D, Lambert J, et al. Acute respiratory failure in kidney transplant recipients: a multicenter study. Crit Care. 2011;15: R91.

19. Mouloudi E, Massa E, Georgiadou E, et al. Course and outcome of renal transplant recipients admitted to the intensive care unit: a 20-year study. Transplant Proc. 2012;44:2718-20.

20. Ting SM, Iqbal $H$, Hamborg $T$, et al. Reduced functional measure of cardiovascular reserve predicts admission to critical care unit following kidney transplantation. PLoS One. 2013;8(5):e64335.

21. Kogan A, Singer P, Cohen J, et al. Readmission to an intensive care unit following liver and kidney transplantation: a 50-month study. Transplant Proc. 1999;31:1892-3.

22. Al-Khafaji A, Huang DT. Critical care management of patients with end-stage liver disease. Crit Care Med. 2011;39:1157-66.

23. DiMartini A, Crone C, Fireman M, Dew MA. Psychiatric aspects of organ transplantation in critical care. Crit Care Clin. 2008;24:949-81.

24. United Network for Organ Sharing: Transplant trends. https:// www.unos.org/data/transplant-trends/\#transplant_by_ organ_type+year+2018

25. Park GR, Gomez-Arnau J, Lindop MJ, Klinck JR, Williams R, Calne RY. Mortality during intensive care after orthotopic liver transplantation. Anaesthesia. 1989;44:959-63.

26. Ramsay M. Justification for Routine Intensive Care After Liver Transplantation. Liver Transpl. 2013;19: S1-5.

27. Lamba S, Murphy P, McVicker S, Harris Smith J, Mosenthal AC. Changing end-of-life care practice for liver transplant service patients: structured palliative care intervention in the surgical intensive care unit. J Pain Symptom Manage. 2012;44:508-19. 